IOS Press

\title{
Erratum
}

\section{Professional Tribute: Jay Neufeld}

Timothy Brei and Amy Houtrow

[Journal of Pediatric Rehabilitation Medicine 10 (3,4) (2017), 159-160]

In this Professional Tribute, it was stated that Dr. Neufeld completed his training in pediatrics and physical medicine and rehabilitation in 1988. The correct year is 1998. We apologize for the error. 